\title{
Attributable Cost and Length of Stay for Central Line-Associated Bloodstream Infections
}

AUTHORS: Anthony Goudie, PhD, ${ }^{a}$ Linda Dynan, PhD, b,c Patrick W. Brady, MD, MSc, ,,d and Mallikarjuna Rettiganti, $\mathrm{PhD}^{\mathrm{e}}$

${ }^{a}$ Center for Applied Research and Evaluation, and ${ }^{~}$ Division of Biostatistics, Department of Pediatrics, University of Arkansas for Medical Sciences, Little Rock, Arkansas; 'bames M. Anderson Center for Health Systems Excellence, and dDivision of Hospital Medicine, Cincinnati Children's Hospital Medical Center, Cincinnati, Ohio; and 'Haile US Bank College of Business, Northern Kentucky University, Highland Heights, Kentucky

KEY WORDS

bloodstream infection, central venous catheter, economic burden, quality of care, cost analysis

\section{ABBREVIATIONS}

AHRQ-Agency for Healthcare Research and Quality

CCS - clinical classification software

CLABSI — central line-associated bloodstream infection

HAl-hospital-acquired infection

HCUP_-Healthcare Cost and Utilization Project

ICD-9-CM-International Classification of Diseases, Ninth

Revision, Clinical Modification

LOS-length of stay

NIS-Nationwide Inpatient Sample

$\mathrm{PDI}$-pediatric quality indicator

POA — present on admission

Dr Goudie conceptualized and designed the study, analyzed and interpreted the data, and drafted and revised the manuscript; Dr Dynan conceptualized and designed the study, interpreted the data, and drafted and revised the manuscript; Dr Brady conceptualized and designed the study, interpreted the data, and revised the manuscript; Dr Rettiganti designed the study, interpreted the data, and revised the manuscript; and all authors approved the manuscript as submitted.

www.pediatrics.org/cgi/doi/10.1542/peds.2013-3795

doi:10.1542/peds.2013-3795

Accepted for publication Mar 14, 2014

Address correspondence to Anthony Goudie, PhD, 1 Children's Way, Little Rock, AR 72202. E-mail: agoudie@uams.edu

PEDIATRICS (ISSN Numbers: Print, 0031-4005; Online, 1098-4275).

Copyright (C) 2014 by the American Academy of Pediatrics

FINANCIAL DISCLOSURE: The authors have indicated they have no financial relationships relevant to this article to disclose.

FUNDING: Dr Goudie received funding for this work from the Marion B. Lyon Revocable Trust, New Scientist Development Award, and is supported by KL2 award 8 KL2 TR000063 and U award 8 UL1 TR000039.

POTENTIAL CONFLICT OF INTEREST: The authors have indicated they have no potential conflicts of interest to disclose.
WHAT'S KNOWN ON THIS SUBJECT: Central line-associated bloodstream infections (CLABSI) are common types of hospitalacquired infections associated with high morbidity and cost. In recent years, quality improvement initiatives have demonstrated how to reduce the incidence of CLABSI.

WHAT THIS STUDY ADDS: This study presents nationally representative estimates of the cost and length of stay attributable to pediatric CLABSI. We make the business case to justify quality improvement prevention initiatives and the adoption of strategies for cost-effective management of CLABSI.

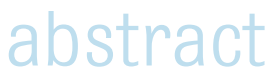

BACKGROUND AND OBJECTIVE: Central line-associated bloodstream infections (CLABSI) are common types of hospital-acquired infections associated with high morbidity. Little is known about the attributable cost and length of stay (LOS) of CLABSI in pediatric inpatient settings. We determined the cost and LOS attributable to pediatric CLABSI from 2008 through 2011.

METHODS: A propensity score-matched case-control study was performed. Children $<18$ years with inpatient discharges in the Nationwide Inpatient Sample databases from the Healthcare Cost and Utilization Project from 2008 to 2011 were included. Discharges with CLABSI were matched to those without CLABSI by age, year, and high dimensional propensity score (obtained from a logistic regression of CLABSI status on patient characteristics and the presence or absence of 262 individual clinical classification software diagnoses). Our main outcome measures were estimated costs obtained from cost-to-charge ratios and LOS for pediatric discharges.

RESULTS: The mean attributable cost and LOS between matched CLABSI cases (1339) and non-CLABSI controls (2678) was \$55646 (2011 dollars) and 19 days, respectively. Between 2008 and 2011, the rate of pediatric CLABSI declined from 1.08 to 0.60 per $1000(P<.001)$. Estimates of mean costs of treating patients with CLABSI declined from $\$ 111852$ to $\$ 98621$ (11.8\%; $P<.001)$ over this period, but cost of treating matched non-CLABSI patients remained constant at $\sim \$ 48000$.

CONCLUSIONS: Despite significant improvement in rates, CLABSI remains a burden on patients, families, and payers. Continued attention to CLABSI-prevention initiatives and lower-cost CLABSI care management strategies to support high-value pediatric care delivery is warranted. Pediatrics 2014;133:e1525-e1532 
Hospital-acquired infections (HAls) harm hospitalized patients and reduce the value of care. Central line-associated bloodstream infections (CLABSIs) are common types of HAls that are associated with particularly high morbidity and cost.1,2 The Joint Commission defines CLABSI as "a primary bloodstream infection (that is, there is no apparent infection at another site) that develops in a patient with a central line in place within the 48-hour period before onset of the bloodstream infection that is not related to infection at another site."3 Published estimates of attributable costs of CLABSI in adult patients have varied over years and across hospital settings,, 45 with 2010 estimates of $\$ 50094$ per event from 1 tertiary care, academic medical center. ${ }^{6} \mathrm{~A}$ pediatric study found an attributable 2008 dollar cost of $\$ 33039$ per CLABSI in a sample of 22 critically ill children. ${ }^{7}$

Quality improvement collaboratives, largely based in ICUs, have demonstrated that most CLABSIs are preventable. Michigan's Keystone ICU project achieved and sustained a $60 \%$ reduction in CLABSI rates in adult ICUs, resulting in 1500 lives saved and $\$ 200$ million in health cost savings over 18 months.8,9 A $43 \%$ reduction was subsequently achieved by a pediatric ICU quality improvement collaborative. ${ }^{10}$

Several government initiatives are intended to reduce the rate of HAls, including CLABSIs. The Department of Health and Human Services oversees 2 of these initiatives: the 2009 HHS Action Plan to Prevent Healthcare-Associated Infections ${ }^{11}$ and the 2011 Partnership for Patients. ${ }^{12}$ These initiatives enlist voluntary participation from providers and hospitals. In addition, government legislation disallowing Medicare (2008) and Medicaid (2012) reimbursement for low-quality care (eg, the costs associated with hospital-acquired CLABSI) is intended to support the high-value objective. Although several studies have found little impact of nonpayment on quality improvement, ${ }^{13,14}$ the use of nonpayment as a policy tool to deter low quality suggests that the excess costs associated with the occurrence of a provider-induced injury or illness are sufficiently high to provide incentives against HAl.

Despite government initiatives to promote the adoption of best-evidence practices to reduce HAls, the variability of HAl rates across hospitals remains high. ${ }^{10}$ Hospitals that do not participate in quality improvement collaboratives to reduce HAls may be unable to replicate the successes of those identified in the literature due to resource, or other, constraints. Missing from the literature are up-to-date, hospital-wide, and nationally representative attributable costs and length of stay (LOS) estimates for the burden of pediatric CLABSI. Understanding the business case for pediatric CLABSI reduction will help hospitals justify needed resources.

We aimed to determine the costs and LOS burden attributable to pediatric CLABSI from 2008 to 2011. Over this period, CLABSI-prevention practices, including those effectively demonstrated in Michigan's Keystone ICU project, should have been widely translated and adopted. In addition, we explored patient-level characteristics associated with pediatric CLABSI. We hypothesized that as adoption continued to spread across hospitals, CLABSI rates would continue to decline between 2008 and 2011, but attributable costs and LOS of pediatric CLABSI, as with adult inpatient populations, ${ }^{15-18}$ would still be substantial and sufficient to target more widespread and intensive CLABSI-prevention initiatives and cost-effective management strategies to support high-value care delivery.

\section{METHODS}

\section{Data Source}

Data were obtained from annual 2008 to 2011 Nationwide Inpatient Sample (NIS) databases from the Healthcare Cost and Utilization Project (HCUP) sponsored by the Agency for Healthcare Research and Quality (AHRQ). The NIS contains complete inpatient discharge information for a representative sample of $\sim 20 \%$ of US community hospitals each year. Discharge- and hospitallevel weights allow for national estimates of event frequency. ${ }^{19}$

\section{Inclusion and Exclusion Criteria}

The study sample consisted of all discharge records for children $<18$ years of age who met criteria for inclusion in the calculation of the pediatric quality indicator (PDI) rate for CLABSI. ${ }^{20}$ These criteria excluded all discharges in which the principal diagnosis was CLABSI, as these were likely non-hospital-acquired CLABSI or CLABSI that was the result of a previous hospital stay. Per AHRQ PDI methods, healthy newborns, neonates with birth weight $<500$ g, visits with an LOS $<2$ days, and pregnancy-related visits were excluded. We further excluded discharges with missing age, gender, or principal diagnosis.

We focused on overall cost and LOS for a complete hospital episode of care; hence, we also excluded all discharges that indicated that the patient died in hospital or transferred in from, or out to, another hospital. We restricted the analysis to discharge records with nonmissing costs and LOS (>99\%).

\section{Main Outcomes}

Estimated inpatient costs and actual LOS were the main outcomes studied. We converted inpatient charges to estimated standardized costs using costto-charge ratio files obtained from HCUP for better comparability because charges poorly represent resource use 
and vary widely across hospitals. Each year of costs was adjusted to 2011 dollars using the Bureau of Labor Statistics' consumer price indices for hospital services between 2008 and $2011 .{ }^{21}$

\section{CLABSI Main Predictor and Other Covariates}

CLABSI diagnosis was the main exposure and predictor of cost and LOS under study. It was defined as any discharge where 1 of 14 secondary diagnoses contained an International Classification of Diseases, Ninth Revision, Clinical Modification (ICD-9-CM) diagnosis code of 999.31 (central venous catheter infection) or 999.32 (bloodstream infection due to central venous catheter). CLABSI rate denominator and numerator designations were compiled by using the AHRQ March 2012 release (Version 4.4) of the PDI SAS software program (http://www. qualityindicators.ahrq.gov/software/SAS. aspx) (SAS Institute, Inc, Cary, NC).

Estimates of hospital inpatient costs and LOS were obtained after adjusting for patient-mix covariates (discharge year, age, gender, race/ethnicity, and insurance status) in all statistical models. AHRQ's PDI SAS program that identifies CLABSI events recodes missing race/ ethnicity values as "Other" for hospitals and states that do not record these data. We also re-categorized Asian or Pacific Islander and Native American children as "Other" because of small category numbers. Insurance status includes categories in which the primary payer is private, Medicaid, uninsured (self-pay), and other. The constructed "Other" insurance category includes discharges covered by Medicare, explicitly listed as no charge or other, and missing.

To account for severity and medical complexity of patients, we derived a propensity score for acquiring a CLABSI using logistic regression where CLABSI (yes/no) was the dependent variable and age, gender, race/ethnicity, insurance status, year of discharge, and dichotomous indicators of clinical classification software (CCS) diagnoses were the covariates. ${ }^{22} \mathrm{CCS}$ is a software tool that aggregates ICD-9-CM diagnoses codes into higher levels of clinical classifications. A single-level CCS classification is included on the NIS for each diagnosis that is listed on the discharge record. For each CCS classification, we created a dichotomous variable (coded as 1 if the classification was listed on the NIS discharge record, 0 otherwise). We included 262 dichotomous non-E-code related CCS covariates in the logistic regression model predicting risk of CLABSI. We did not include the CCS category that included the CLABSI diagnosis.

\section{Statistical Analyses}

In the descriptive analysis, we calculated the weighted incidence and rate of CLABSI by patient characteristic categories. Based on a logistic regression in which CLABSI status is regressed on patient characteristics and dichotomous clinical classifications, we also present the adjusted odds ratios (with 95\% confidence intervals) for CLABSI by comparing patient characteristics across categories and the median (with interquartile range) adjusted propensity scores. To demonstrate that certain clinical classifications are highly predictive of CLABSI, we present the 10 most significant with an incidence of at least 10 in the study population.

Each CLABSI case was matched with 2 controls (no CLABSI) by age group, year, and propensity score by using a greedy matching algorithm. ${ }^{23}$ We successfully matched 2 controls for 1339 (98.6\%) of 1358 CLABSI cases. The 19 unmatched cases were uniquely characterized with multiple comorbid diagnoses and a very high risk for CLABSI. Of those matched, the mean relative percentage difference in propensity score between cases and controls was $<1.0 \%$ (data not shown). The matching of discharges with an indication of CLABSI to those without and by using an appropriate statistical analytical technique to account for the cost and LOS distributions reduce potential biases in least-squares means results.

A generalized linear mixed model (PROC GLIMMIX in SAS/STAT software) was used to estimate and compare costs and LOS between CLABSI cases and matched controls. A Gamma distribution with a log link function was used to model cost, whereas LOS was modeled by using a negative binomial distribution. The CLABSI (yes/no) indicator was the main predictor. Other covariates included age, gender, race/ethnicity, insurance, year, propensity score (to account for variability across the matched groups), and interactions for CLABSI by age, gender, race/ethnicity, insurance category, and year. Matching of case and controls was taken into account by introducing a random effect for the matching identifier. Leastsquares estimates and $P$ values were obtained from the fitted models.

To evaluate the association between propensity score and resource use, we profiled estimated adjusted mean costs and LOS by CLABSI status within quintiles of increasing propensity score values. To achieve this, we substituted the continuous propensity score with the associated propensity score quintile variable in the case-control adjusted model and calculated the least squares means for costs and LOS by CLABSI status and propensity score quintile.

Descriptive analyses included population weights and hospital strata to account for the NIS sampling design. All analyses were performed by using SAS Enterprise Guide 4.3 (SAS Institute, Inc). All statistical tests were evaluated with a 2-sided $5 \%$ significance level. 


\section{RESULTS}

In the combined 4-year analytical dataset of children at risk for CLABSI, we analyzed 1670140 pediatric inpatient discharge records representing 8215 758 population-weighted discharges. Hospital-acquired CLABSI was listed on 1358 discharge records, representing an estimated 6708 cases.

Pediatric CLABSI rates varied across age, gender, race/ethnicity, and insurance status (Table 1). Children aged 1 to 4 years, boys, African American children, and those with an insurance status of "Other" were disproportionately more likely to experience a CLABSI within each patient characteristic. In general, higher median propensity scores were associated with higher adjusted odds of acquiring a CLABSI. A notable exception is where African American children have a lower score than Hispanic children, but have higher adjusted odds and rate. A number of clinical classifications (many cancerrelated) reflective of the acuity of the condition and comorbidities are significantly associated with CLABSI. Consistent with high acuity and comorbidity, children with these conditions are a priori at higher risk for CLABSI and are more likely to experience CLABSI.

Across all categories of age, gender, race/ethnicity, insurance status, and year of discharge, the adjusted mean attributable cost and LOS difference for children acquiring a CLABSI was significantly higher $(P<.05)$ than similar children not acquiring a CLABSI (Tables 2 and 3). Overall, a CLABSI was associated with a mean attributable cost of $\$ 55646$ and attributable LOS of 19 days (both $P<$.001). A CLABSI experienced by a neonate was associated with a mean attributable cost difference of $\$ 90221$ and LOS difference of 31.5 days compared with matched neonates who did not acquire a CLABSI. Uninsured patients who acquired a CLABSI had higher mean attributable costs (\$83 409) than privately insured patients (\$41 990; $P<.05$ ), despite having lower overall rates and risk of CLABSI. A CLABSI acquired by an uninsured child was associated with a mean attributable LOS of approximately 4 weeks (28.8 days; $P<.05$ ).

TABLE 1 Adjusted CLABSI Incidence, Rate, and Adjusted Risk of CLABSI by Child Demographics, Insurance, and Year of Inpatient Discharge Categories and Clinical Conditions, 2008-2011

\begin{tabular}{|c|c|c|c|c|c|}
\hline Patient Characteristics & Category & Weighted $n$ with CLABSI & Actual Rate per 1000 & AOR $(95 \% \mathrm{Cl})$ & Propensity Score $\times 1000$, Median (IQR) \\
\hline \multirow[t]{5}{*}{ Age, y } & Neonate & 1336 & 0.351 & $0.80(0.70-0.93)$ & $0.104(0.054-0.214)$ \\
\hline & $<1$ & 1002 & 0.887 & $1.02(0.91-1.14)$ & $0.217(0.110-0.469)$ \\
\hline & $1-4$ & 1916 & 1.737 & $1.62(1.48-1.77)$ & $0.326(0.147-0.741)$ \\
\hline & $5-12$ & 1427 & 1.258 & $1.23(1.13-1.34)$ & $0.200(0.093-0.484)$ \\
\hline & $13-17$ & 1027 & 0.983 & reference & $0.143(0.057-0.355)$ \\
\hline \multirow[t]{2}{*}{ Gender } & Male & 3863 & 0.873 & $1.17(1.11-1.23)$ & $0.161(0.075-0.369)$ \\
\hline & Female & 2845 & 0.750 & reference & $0.140(0.064-0.323)$ \\
\hline \multirow[t]{4}{*}{ Race/ethnicity } & White & 2733 & 0.779 & reference & $0.150(0.070-0.336)$ \\
\hline & Black & 1085 & 0.897 & $1.33(1.23-1.43)$ & $0.173(0.079-0.411)$ \\
\hline & Hispanic & 1708 & 1.122 & $1.29(1.21-1.38)$ & $0.182(0.084-0.429)$ \\
\hline & Other & 1183 & 0.599 & $0.88(0.82-0.94)$ & $0.123(0.058-0.273)$ \\
\hline \multirow[t]{4}{*}{ Insurance } & Private & 2440 & 0.692 & reference & $0.137(0.065-0.303)$ \\
\hline & Public & 3752 & 0.924 & $1.15(1.09-1.21)$ & $0.169(0.078-0.394)$ \\
\hline & Uninsured & 76 & 0.254 & $0.62(0.49-0.77)$ & $0.072(0.035-0.150)$ \\
\hline & Other & 441 & 1.344 & $1.35(1.22-1.50)$ & $0.209(0.095-0.515)$ \\
\hline \multirow[t]{4}{*}{ Year } & 2008 & 2201 & 1.084 & reference & $0.241(0.116-0.511)$ \\
\hline & 2009 & 1384 & 0.670 & $0.59(0.55-0.64)$ & $0.137(0.066-0.292)$ \\
\hline & 2010 & 1983 & 0.892 & $0.65(0.61-0.69)$ & $0.160(0.076-0.364)$ \\
\hline & 2011 & 1140 & 0.600 & $0.43(0.40-0.46)$ & $0.096(0.045-0.216)$ \\
\hline Overall & & 6708 & 0.817 & & $0.151(0.070-0.347)$ \\
\hline \multicolumn{6}{|c|}{ Clinical classifications (referent class does not have condition) } \\
\hline \multicolumn{3}{|c|}{ Other injuries and conditions due to external causes } & & $5.91(5.59-6.32)$ & $3.236(1.761-6.063)$ \\
\hline \multicolumn{3}{|c|}{ Cancer; other and unspecified primary } & & $5.52(4.78-6.40)$ & $3.396(1.782-7.595)$ \\
\hline \multicolumn{3}{|l|}{ Leukemias } & & $5.04(4.58-5.54)$ & $3.116(1.543-8.424)$ \\
\hline \multicolumn{3}{|c|}{ Respiratory distress syndrome } & & $4.75(4.23-5.35)$ & $1.220(0.505-3.038)$ \\
\hline \multicolumn{3}{|c|}{ Phlebitis; thrombophlebitis and thromboembolism } & & $4.44(4.00-4.93)$ & $4.604(1.306-16.112)$ \\
\hline \multicolumn{3}{|c|}{ Cancer of other gastrointestinal organs; peritoneum } & & $4.06(2.84-5.79)$ & $3.129(1.384-8.418)$ \\
\hline \multicolumn{3}{|l|}{ Burns } & & $3.72(2.80-4.94)$ & $0.655(0.425-1.156)$ \\
\hline \multicolumn{3}{|l|}{ Mycoses } & & $3.72(3.45-4.00)$ & $1.376(0.563-4.558)$ \\
\hline \multicolumn{3}{|c|}{ Cancer of brain and nervous system } & & $3.70(3.14-4.35)$ & $1.915(0.917-4.640)$ \\
\hline \multicolumn{3}{|c|}{ Regional enteritis and ulcerative colitis } & & $3.60(3.14-4.34)$ & $1.036(0.513-2.481)$ \\
\hline
\end{tabular}

Neonates are children born in the hospital with complications. The median propensity score by variable category is the point where one-half of discharges associated with the category have a lower, and one-half have a higher probability of incurring a CLABSI.

$\mathrm{Cl}$, confidence interval; AOR, unadjusted odds ratio with CLABSI (1-yes, 0-no) as the logistic regression outcome; IQR, interquartile range.

Source: Nationwide Inpatient Sample, 2008-2011. 
TABLE 2 Estimated Cost (in 2011 Dollars) With 95\% Confidence Intervals by CLABSI and Patient Characteristics

\begin{tabular}{|c|c|c|c|c|c|}
\hline \multirow[t]{2}{*}{ Patient Characteristics } & \multirow[t]{2}{*}{ Category } & \multicolumn{2}{|c|}{ Estimated Cost } & \multirow{2}{*}{$\frac{\text { Difference }}{\text { (95\% Confidence Interval) }}$} & \multirow{2}{*}{$\frac{\text { Relative Difference Ratio }}{\text { (95\% Confidence Interval) }}$} \\
\hline & & CLABSI & No CLABSI & & \\
\hline \multirow[t]{5}{*}{ Age, y } & Neonate & $206982^{a}$ & $116761^{\mathrm{a}}$ & 90221 (45 036-135 406) & $1.77(1.37-2.37)$ \\
\hline & $<1$ & 116862 & $57168^{\mathrm{b}}$ & $59693(33667-85719)$ & $2.04(1.46-2.80)$ \\
\hline & $1-4$ & $70374^{\mathrm{a}}$ & $30554^{\mathrm{a}}$ & 39820 (26 944-52 696) & $2.30(1.73-3.02)$ \\
\hline & $5-12$ & $76429^{\mathrm{a}}$ & $33871^{\mathrm{a}}$ & 42558 (27 800-57 316) & $2.26(1.67-2.99)$ \\
\hline & $13-17$ & 93288 & $38064^{\mathrm{a}}$ & 55224 (36 092-74 356) & $2.45(1.76-3.33)$ \\
\hline \multirow[t]{2}{*}{ Gender } & Male & 102898 & 49356 & 52505 (34 486-70 524) & $2.04(1.58-2.62)$ \\
\hline & Female & 105011 & 42725 & 58711 (40 752-76 670) & $2.27(1.74-2.92)$ \\
\hline \multirow[t]{4}{*}{ Race/ ethnicity } & White & 95248 & 45312 & 49936 (32 948-66 924) & $2.10(1.61-2.72)$ \\
\hline & Black & 103224 & 47010 & 56214 (34 267-78 161) & $2.20(1.58-2.98)$ \\
\hline & Hispanic & 108703 & $53161^{b}$ & 55542 (34 022-77 063) & $2.05(1.52-2.71)$ \\
\hline & Other & 109246 & 48073 & 61173 (39 451-82 895) & $2.27(1.67-3.04)$ \\
\hline \multirow[t]{4}{*}{ Insurance } & Private & 93869 & 51879 & 41990 (31 278-52 702) & $1.81(1.54-2.11)$ \\
\hline & Public & 105970 & 51465 & 54505 (45 230-63 780) & $2.06(1.81-2.33)$ \\
\hline & Uninsured & 124505 & 41096 & 83409 (4550-162 268) & $3.03(0.98-8.41)$ \\
\hline & Other & 94273 & 49612 & 44661 (22 066-67 256) & $1.90(1.32-2.62)$ \\
\hline \multirow[t]{4}{*}{ Year } & 2008 & 111852 & 48369 & 63483 (43 567-83 399) & $2.31(1.75-3.01)$ \\
\hline & 2009 & 112588 & $54112^{b}$ & 58476 (35 046-81 906) & $2.08(1.52-2.79)$ \\
\hline & 2010 & 94011 & $43431^{b}$ & 50580 (33 147-68 013) & $2.17(1.63-2.83)$ \\
\hline & 2011 & 98621 & 47888 & 50733 (30 523-70 943) & $2.06(1.51-2.75)$ \\
\hline Overall & & 103949 & 48303 & $55646(38785-72507)^{\mathrm{C}}$ & $2.15(1.69-2.72)^{\mathrm{C}}$ \\
\hline
\end{tabular}

Estimated costs are obtained from a well-fit $(P<.001)$ generalized linear mixed model regression with propensity score $(P<.001)$, CLABSI status $(P<.001)$, age $(P<.001)$, and year $(P<.05)$ as significant predictors. Gender, race/ethnicity, insurance status, and CLABSI status by age, gender, race/ethnicity, insurance, and year interactions were also included in the model but not significant predictors of cost.

Estimated cost for Difference $=$ CLABSI - No CLABSI and Relative Difference Ratio $=$ CLABSI $\div$ № CLABSI .

Source: Nationwide Inpatient Sample, 2008-2011.

a Significant difference between patient characteristic category and mean of other categories at $P<.001$.

b Significant difference between patient characteristic category and mean of other categories at $P<.05$.

c Significant overall difference at $P<.001$.

TABLE 3 Estimated Adjusted Mean LOS (in Days) by CLABSI and Patient Characteristics

\begin{tabular}{|c|c|c|c|c|c|}
\hline \multirow[t]{2}{*}{ Patient Characteristics } & \multirow[t]{2}{*}{ Category } & \multicolumn{2}{|c|}{ Estimated LOS } & \multirow{2}{*}{$\frac{\text { Difference }}{(95 \% \text { Confidence Interval) }}$} & \multirow{2}{*}{$\frac{\text { Relative Difference Ratio }}{\text { (95\% Confidence Interval) }}$} \\
\hline & & CLABSI & No CLABSI & & \\
\hline \multirow[t]{5}{*}{ Age, y } & Neonate & $91.8^{\mathrm{a}}$ & $60.2^{\mathrm{a}}$ & $31.5(14.9-48.1)$ & $1.52(1.21-1.90)$ \\
\hline & $<1$ & $42.6^{b}$ & $23.3^{\mathrm{a}}$ & $19.4(11.7-27.0)$ & $1.83(1.42-2.33)$ \\
\hline & $1-4$ & $27.0^{\mathrm{a}}$ & $10.7^{\mathrm{a}}$ & $16.3(12.6-20.0)$ & $2.53(2.04-3.12)$ \\
\hline & $5-12$ & $24.1^{\mathrm{a}}$ & $11.0^{\mathrm{a}}$ & $13.2(9.5-16.8)$ & $2.20(1.75-2.74)$ \\
\hline & $13-17$ & $27.9^{\mathrm{a}}$ & $12.1^{\mathrm{a}}$ & $15.8(11.3-20.3)$ & $2.30(1.80-2.92)$ \\
\hline \multirow[t]{2}{*}{ Gender } & Male & 36.1 & 18.5 & $17.6(12.6-22.5)$ & $1.95(1.60-2.36)$ \\
\hline & Female & 38.3 & 17.9 & $18.2(13.3-23.1)$ & $2.02(1.65-2.45)$ \\
\hline \multirow[t]{4}{*}{ Race/ ethnicity } & White & 35.6 & 17.1 & $18.5(13.6-23.4)$ & $2.08(1.70-2.54)$ \\
\hline & Black & 41.0 & 19.2 & $21.7(15.0-28.5)$ & $2.13(1.66-2.70)$ \\
\hline & Hispanic & 35.4 & 18.5 & $16.9(11.3-22.4)$ & $1.91(1.53-2.38)$ \\
\hline & Other & 37.1 & 18.0 & $19.1(13.2-25.0)$ & $2.06(1.63-2.58)$ \\
\hline \multirow[t]{4}{*}{ Insurance } & Private & $31.4^{\mathrm{b}}$ & 18.5 & $12.9(10.1-15.7)$ & $1.70(1.50-1.91)$ \\
\hline & Public & 38.8 & 19.4 & $19.4(16.7-22.0)$ & $2.00(1.81-2.20)$ \\
\hline & Uninsured & 46.0 & 17.2 & $28.8(6.1-51.6)$ & $2.67(1.23-5.61)$ \\
\hline & Other & 34.1 & $17.6^{\mathrm{a}}$ & $16.5(10.2-22.7)$ & $1.93(1.47-2.48)$ \\
\hline \multirow[t]{4}{*}{ Year } & 2008 & $40.5^{b}$ & 18.5 & $22.1(16.4-27.7)$ & $2.19(1.78-2.69)$ \\
\hline & 2009 & 36.6 & 19.3 & $17.3(11.2-23.4)$ & $1.89(1.49-2.38)$ \\
\hline & 2010 & 35.9 & $16.4^{\mathrm{b}}$ & $19.4(14.3-24.6)$ & $2.18(1.76-2.69)$ \\
\hline & 2011 & 36.0 & 18.7 & $17.3(11.4-23.2)$ & $1.93(1.53-2.41)$ \\
\hline Overall & & 37.2 & 18.2 & $19.0(14.3-23.8)^{\mathrm{C}}$ & $2.05(1.70-2.60)^{\mathrm{C}}$ \\
\hline
\end{tabular}

Estimated LOS is obtained from a well-fit $(P<.001)$ generalized linear mixed model regression with propensity score $(P<.001)$, CLABSI status $(P<.001)$, age $(P<.001)$, CLABSI status by age $(P<.001)$, race/ethnicity $(P<.05)$, insurance status $(P<.001)$, and year $(P<.05)$ as significant predictors. Gender and CLABSI status by gender, race/ethnicity, insurance, and year interactions were also included in the model but not significant predictors of LOS.

Estimated LOS for Difference $=$ CLABSI - No CLABSI and Relative Difference Ratio $=$ CLABSI $\div$ No CLABSI.

Source: Nationwide Inpatient Sample, 2008-2011.

a Significant difference between patient characteristic category and mean of other categories at $P<.001$.

b Significant difference between patient characteristic category and mean of other categories at $P<.05$.

${ }^{c}$ Significant overall difference at $P<.001$. 
Between 2008 and 2010, mean adjusted cost estimates for patients with a CLABSI declined from $\$ 111852$ to $\$ 94$ 011, but increased slightly again in 2011 to $\$ 98621$ (economically but not statistically significant due to high variability). For patients who do not acquire CLABSI, mean adjusted cost estimates did not decline between 2008 (\$48 369) and 2011 (\$47888). Similar results are noted for estimated mean LOS.

Table 4 presents mean adjusted cost and LOS estimates for discharges associated with each quintile of increasing propensity score. In general, estimated attributable costs and LOS differences do not increase uniformly as propensity score quintile increases; however, relative difference ratios are consistently higher in lower propensity score quintiles. Costs associated with inpatient stays for children with CLABSI are 3.73 times higher (relative difference ratio) for children in the lowest quintile than for children in the same propensity score percentile who do not acquire CLABSI. This ratio drops to 1.35 for children in the highest quintile. A similar trend is observed for LOS.

\section{DISCUSSION}

Our estimated cost and LOS differences attributable to pediatric CLABSI indicate

TABLE 4 Estimated Costs (in 2011 Dollars) and LOS (in Days) by Propensity Score Quintile

\begin{tabular}{ccccc}
\hline & CLABSI & No CLABSI & $\begin{array}{c}\text { Difference } \\
\text { (95\% Confidence Interval) }\end{array}$ & $\begin{array}{c}\text { Relative Difference Ratio } \\
\text { (95\% Confidence Interval) }\end{array}$ \\
\hline $\begin{array}{c}\text { Estimated cost } \\
\text { Risk quintile }\end{array}$ & & & & \\
1 & 58106 & 15581 & $42525(32967-52083)$ & $3.73(2.80-4.88)$ \\
2 & 88909 & 28551 & $60358(45970-74746)$ & $3.14(2.37-4.04)$ \\
3 & 96267 & 43401 & $52866(35218-70514)$ & $2.22(1.67-2.89)$ \\
4 & 117845 & 75089 & $42747(17621-67873)$ & $1.57(1.18-2.05)$ \\
5 & 168976 & 124914 & $44062(4050-84074)$ & $1.35(1.01-1.78)$ \\
Estimated LOS & & & & \\
Risk quintile & & & $14.1(11.2-17.0)$ & $2.90(2.32-3.59)$ \\
1 & 21.5 & 7.4 & $18.7(14.7-22.8)$ & $2.59(2.09-3.17)$ \\
2 & 30.5 & 11.8 & $19.8(14.4-25.1)$ & $2.15(1.73-2.65)$ \\
3 & 37.0 & 17.2 & $17.5(10.5-24.5)$ & $1.67(1.35-2.06)$ \\
4 & 43.4 & 26.0 & $17.2(6.9-27.6)$ & $1.43(1.14-1.77)$ \\
\hline
\end{tabular}

that large potential cost savings associated with decreased CLABSI rates and better CLABSI care management exist. We estimated a mean attributable cost of $\$ 55646$ (Table 2) and increased LOS of 19 days.

Cost savings potential are greatest among children in lower propensity score quintiles. This is most evident viewing relative difference ratios. Hospitals may find it beneficial to target quality improvement efforts and vigilance toward understanding and implementing CLABSI prevention in lower quintiles of the propensity score distribution. Reducing CLABSI in children who would otherwise have short LOS not only saves more money, but also creates more turnover for revenuegenerating inpatient "first days" (assuming hospitals staff beds based on full census). ${ }^{7}$ Along with prevention, we note potential saving in cost from the care management of CLABSI. There was a decrease in estimated cost of $11.8 \%$ (\$111 852 in 2008 to $\$ 98621$ in 2011) of treating CLABSI but a negligible decrease in the cost of treating patients who do not acquire CLABSI over the same time.

Our findings identify 2 patient groups to flag for increased attention to CLABSI prevention vigilance: neonates and uninsured patients. Neonates and uninsured patients have low risk and low rates of CLABSI, but have high treatment cost and LOS when CLABSI is acquired. Likewise, strategies for improved management of these patients if they acquire CLABSI suggest potential cost savings. In addition, understanding the higher risk associated with 1- to 4-yearolds and targeting improvement initiatives toward this group may reduce the burden of CLABSI and have spillover effects to all patients at risk.

We found that CLABSI rates can be reduced, not only in ICU settings, as previously reported, but also in a nationally representative sample that includes children outside of the ICU who are at risk for CLABSI. We observed a $45 \%$ overall reduction in CLABSIs over our 4-year study (1.084 per 1000 in 2008 to 0.600 per 1000 in 2011), similar to the $43 \%$ reduction found in the largest pediatric improvement collaborative but below the $60 \%$ reduction attained in the Keystone ICU project. This decrease also suggests that significant reductions in CLABSIs are possible without participation in a quality improvement collaborative network or real-time infection data that were identified as potential sources for the success of the Keystone project. ${ }^{24}$

On average, we found that a CLABSI cost \$55 646 (in 2011 dollars) and prolonged LOS by 19 days. Differences in estimates between previous studies and ours are likely not only because of different populations and study years, but also because of different methods for matching patients with CLABSI to those who do not acquire CLABSI. The use of matching by propensity score allows for more precise comparison between patients where estimated differences in cost and LOS are due to CLABSI. To our knowledge, the only study to estimate cost of hospital-acquired bloodstream infections (a somewhat different event) by using a matched case-control methodology (by age, gender, and primary diagnosis on admission, but not 
propensity score) found a cost of \$62 992 per infection in an adult NIS sample from 2003. ${ }^{2}$

Our study has limitations. NIS does not have central-line days, precluding our ability to identify the portion of decreased risk associated with decreased centralline days per discharge. We nonetheless believe that the reductions in CLABSI rates per discharge represent an important and meaningful rate for clinicians, researchers, and policymakers.

Conditions present on admission (POA) are represented in the HCUP NIS only beginning in the fourth quarter of 2008. Even after this, the recording of POA is not universal across hospitals and states. We attempted to control for CLABSI POA by excluding discharges where CLABSI was listed as a primary diagnosis and/or indicated the patient transferred in from another hospital setting. The possibility of misclassification in administrative data, particularly underidentification of CLABSls through ICD-9-CM diagnosis codes, is an additional limitation. One study suggests this may be particularly important with estimates of neonatal CLABSI rate. ${ }^{25}$ We limited the number of controls that were matched to each discharge case with a CLABSI to 2 to maximize the percentage of CLABSI cases matched to non-CLABSI controls. The 19 cases that were unmatched are mostly associated with very high risk for CLABSI and very high costs and LOS (data not shown).

Future research is needed to identify how (internal procedures, processes, and policies) many hospitals are succeeding in lowering pediatric CLABSI risk and incidence while others continue to struggle. A rigorous trend analysis will help identify the heterogeneity of CLABSI prevention and treatment management across hospitals. More specific research is also warranted to look at the types of procedures performed that lead to CLABSI and how the adoption of newer central line devices, and technology in general, affect CLABSI rates.

\section{CONCLUSIONS}

Ongoing payment reform initiatives across all payers, such as moving from fee-for-service to prospective payment, claim denial for poor-quality care (including hospital-acquired CLABSI), and bundled payment for episodes of care will shift more of the burden of attributable costs of CLABSI back to hospitals. We have demonstrated a business case for the adoption of best-evidence practices to prevent, and, when necessary, better manage pediatric CLABSI.

\section{REFERENCES}

1. Srinivasan A, Wise M, Bell M, et al; Centers for Disease Control and Prevention (CDC). Vital signs: central line-associated blood stream infections-United States, 2001, 2008, and 2009. MMWR Morb Mortal Wkly Rep. 2011;60(8):243-248

2. Al-Rawajfah OM, Hewitt JB, Stetzer F, Cheema J. Length of stay and charges associated with health care-acquired bloodstream infections. Am J Infect Control. 2012;40(3):227-232

3. The Joint Commission. Preventing Central Line-Associated Bloodstream Infections: A Global Challenge, a Global Perspective. Oak Brook, IL: Joint Commission Resources, May 2012. Available at: www.PreventingCLABSIs. pdf. Accessed April 16, 2013

4. Shannon RP, Patel B, Cummins D, Shannon AH, Ganguli G, Lu Y. Economics of central line-associated bloodstream infections. Am J Med Qual. 2006;21(suppl 6):7S-16S

5. Warren DK, Quadir WW, Hollenbeak CS, Elward AM, Cox MJ, Fraser VJ. Attributable cost of catheter-associated bloodstream infections among intensive care patients in a nonteaching hospital. Crit Care Med. 2006;34(8):2084-2089

6. Stevens V, Geiger K, Concannon C, Nelson RE, Brown J, Dumyati G. Inpatient costs, mor- tality, and 30-day readmission in patients with central line-associated blood stream infections (CLABSI) [published online ahead of print September 25, 2013]. Clin Microbiol Infect. doi: 10.1111/1469-0691.12407

7. Nowak JE, Brilli RJ, Lake MR, et al. Reducing catheter-associated bloodstream infections in the pediatric intensive care unit: business case for quality improvement. Pediatr Crit Care Med. 2010;11(5):579-587

8. Pronovost P, Needham D, Berenholtz S, et al. An intervention to decrease catheterrelated bloodstream infections in the ICU. N Engl J Med. 2006;355(26):2725-2732

9. I0M (Institute of Medicine). The Richard \& Hinda Rosenthal Lecture 2011: New Frontiers in Patient Safety. Washington, DC: The National Academies Press; 2011

10. Miller MR, Griswold M, Harris JM II, et al. Decreasing PICU catheter-associated bloodstream infections: NACHRI's quality transformation efforts. Pediatrics. 2010;125(2): 206-213

11. Department of Health and Human Services. Action plan to prevent healthcare-associated infections: July 2009 final. Available at: www. hhs.gov/ash/initiatives/hai/actionplan/index. html. Accessed April 18, 2013
12. Department of Health and Human Services. Partnership for patients to improve care and lower costs for Americans. 2011. Available at: www.hhs.gov/news/press/2011pres/ 04/20110412a.html. Accessed April 18, 2013

13. Conrad DA, Perry L. Quality-based financial incentives in health care: can we improve quality by paying for it? Annu Rev Public Health. 2009;30:357-371

14. Lee GM, Hartmann CW, Graham D, et al Perceived impact of the Medicare policy to adjust payment for health care-associated infections. Am J Infect Control. 2012;40(4) 314-319

15. Tarricone R, Torbica A, Franzetti F, Rosenthal VD. Hospital costs of central line-associated bloodstream infections and cost-effectiveness of closed vs. open infusion containers. The case of Intensive Care Units in Italy. Cost Eff Resour Alloc. 2010;8:8

16. Waters HR, Korn R Jr, Colantuoni E, et al The business case for quality: economic analysis of the Michigan Keystone Patient Safety Program in ICUs. Am J Med Qual. 2011;26(5):333-339

17. Crnich CJ. Estimating excess length of stay due to central line-associated bloodstream infection: separating the wheat from the 
chaff. Infect Control Hosp Epidemiol. 2010; 31(11):1115-1117

18. Roberts RR, Scott RD II, Hota B, et al. Costs attributable to healthcare-acquired infection in hospitalized adults and a comparison of economic methods. Med Care 2010;48(11):1026-1035

19. HCUP Nationwide Inpatient Sample (NIS). Healthcare Cost and Utilization Project (HCUP). 2008-2011. Agency for Healthcare Research and Quality, Rockville, MD. Available at: www.hcup-us-ahrq.gov/nisoverview. jsp. Accessed April 16, 2013
20. Agency for Healthcare Research and Quality. Central venous catheter-related blood stream infection rate: technical specifications. Pediatric quality indicators. 2013. Available at: www.qualityindicators.ahrq. gov. Accessed June 9, 2013

21. Bureau of Labor Statistics. Consumer price index. Available at: www.bls.gov/cpi/. Accessed August 14, 2012

22. Elixhauser A, Steiner C, Palmer L. Clinical Classification Software (CCS). Rockville, MD: Agency for Healthcare Research and Quality; 2013
23. Parsons L. Performing a 1:N Case-Control Match on Propensity Score. SAS Users Group International. Montreal, Canada: SAS Institute Inc.; 2004

24. Dixon-Woods M, Bosk CL, Aveling EL, Goeschel CA, Pronovost PJ. Explaining Michigan: developing an ex post theory of a quality improvement program. Milbank Q. 2011;89(2):167-205

25. Patrick SW, Davis MM, Sedman AB, et al. Accuracy of hospital administrative data in reporting central line-associated bloodstream infections in newborns. Pediatrics. 2013;131(suppl 1):S75-S80 